\title{
The preservation of phenotype and functionality of dendritic cells upon phagocytosis of polyelectrolyte-coated PLGA microparticles
}

\author{
Stefan Fischer ${ }^{\mathrm{a}}$, Edith Uetz-von Allmen ${ }^{\mathrm{b}, 1}$, Ying Waeckerle-Men ${ }^{\mathrm{b}, 2}$, Marcus Groettrup ${ }^{\mathrm{c}}$, \\ Hans P. Merkle ${ }^{a}$, Bruno Gander ${ }^{\mathrm{a}, *}$ \\ ${ }^{a}$ Institute of Pharmaceutical Sciences, ETH Zurich, Wolfgang-Pauli Strasse 10, CH-8093 Zurich, Switzerland \\ ${ }^{\mathrm{b}}$ Research Department, Cantonal Hospital St. Gallen, CH-9007 St. Gallen, Switzerland \\ ${ }^{c}$ Department of Biology/Immunology, University of Konstanz, Universitätsstrasse 10, D-78457 Konstanz, Germany
}

\begin{abstract}
Biodegradable microparticles (MP) represent a promising and efficient delivery system for parenteral vaccination. Recently, MP have also been explored as tool for the ex vivo antigen loading of professional antigen-presenting cells such as dendritic cells (DC) to be used as cellular vaccines. The purpose of this study was to investigate various polycationic coatings on poly(lactide-co-glycolide) (PLGA) MP, with regard to their effect on phenotypic and functional maturation of monocyte-derived DC (MoDC) that had previously been loaded with the MP in vitro. The preparation and concomitant coating of the PLGA was performed by means of a solvent extraction/ evaporation method using a recently developed microextrusion-based technique. The polyelectrolytes tested for MP coating encompassed aminodextran, chitosan, poly(ethylene imine) (PEI), poly(L-lysine) and protamine. Uncoated and differently coated PLGA MP were fed to immature MoDC, which ingested efficiently the different MP types irrespective of their surface coating. The MPloaded immature MoDC were then matured with the help of a cytokine/PGE-2 maturation cocktail. Here, the presence of the ingested MP did not affect the MoDC maturation in terms of expression of the surface markers CD80, CD83, CD86, HLA-DR and MMR, irrespective of the MP surface coating. Importantly, none of the PLGA MP types alone induced significant maturation of MoDC in the absence of the maturation cocktail. MP-loaded and subsequently matured MoDC expressed high levels of the chemokine receptor CCR7, whose functional activity was evidenced by the migration of MoDC towards CCL21, irrespective of the presence of ingested MP. Further, MP-loaded and subsequently matured MoDC also secreted comparable amounts of IL-10 and IL-12p70, irrespective of the presence of ingested MP except for PEI-coated PLGA MP, which enhanced significantly the secretion of IL-12p70 in mature MoDC. In conclusion, phenotypic and functional maturation of MoDC by means of a maturation cocktail remained unchanged irrespective of the presence of previously ingested differently coated PLGA MP. This offers interesting perspectives for using these particulate systems together with entrapped antigens for ex vivo loading of MoDC in view of cellular immunotherapy.
\end{abstract}

Keywords: Dendritic cells; Immunomodulation; Microsphere

\section{Introduction}

Biodegradable microparticles (MP) made of poly(lactide-co-glycolide) (PLGA) have been frequently proposed

\footnotetext{
*Corresponding author. Tel.: + 414463373 12; fax: +41446331314.

E-mail address: bruno.gander@pharma.ethz.ch (B. Gander).

${ }^{1}$ Present address: Biotechnology Institute Thurgau (BITg), CH-8274 Tägerwilen, Switzerland.

${ }^{2}$ Present address: Institute of Physiology, University of Zurich, Winterthurerstrasse 190, CH-8057 Zurich, Switzerland.
}

as drug and antigen delivery systems [1-3]. For this use, the particles possess very attractive features that encompass, amongst others, the controlled release of microencapsulated compounds and their rapid and efficient phagocytosis by professional antigen-presenting cells (APC), such as macrophages [4] and dendritic cells (DC), both in vitro [5,6] and in vivo [7]. The uptake of PLGA MP by APC is certainly one of the important elements of the immunological adjuvancy of this particle type. Another element is probably the traveling of phagocytosed particles inside 
APC from the site of injection to draining lymph nodes, the principal sites of antigen presentation [8].

The potential of PLGA MP for antigen delivery has been highlighted by several in vivo studies showing high antibody titers after single administration of antigen loaded MP $[9,10]$. In addition, PLGA MP have also been shown to induce cellular responses, such as CTL activity [11-14]. Finally, PLGA MP have been recognized to serve as intracellular antigen reservoirs in APC after particle uptake so that encapsulated and intracellularly released antigens can be presented by the APC to specific T cells over extended periods of time, e.g., for up to 9 days [15].

Taken together, PLGA MP are nowadays well recognized as potent immuno-adjuvants [16]. The known modes of action encompass (i) phagocytosis by APC, (ii) subsequent migration to the lymph nodes, (iii) intracellular antigen release, and (iv) enhanced antigen presentation inducing both humoral and cellular immune responses.

A certain drawback of PLGA MP is their lack of suitable functional groups for efficient covalent bioconjugation of bioactive ligands or the adsorption of negatively charged therapeutics such as DNA. To overcome this limitation, we recently proposed a novel technology for a one-step preparation of chitosan-coated PLGA MP and their functionalization with ligands [17]. Two features of such chitosan-coated PLGA MP for antigen delivery appear to be of particular interest. Firstly, chitosan coatings exhibit an important fraction of primary amino groups to the environment; these amino groups are freely accessible for a variety of mild coupling reactions for bioactive ligands. The decoration of MP with specific ligands for APC targeting may be used to elicit more specific or enhanced immune responses, e.g., through activation of immature DC [18]. Secondly, the positive charge of chitosan coating likely promotes the ionic binding of nucleic acids, either for gene vaccination purposes, e.g., plasmid DNA or mRNA $[19,20]$, or to deliver an immunoadjuvant, e.g., single-stranded RNA [21] or the doublestranded poly(I:C).

DC play a key role in inducing acquired and innate immunity [22]. Their physiologic role in capturing antigens, whole microbes, viruses, or apoptotic bodies finds extension in their capacity to ingest synthetic microparticulate matter, such as PLGA MP $[23,24]$. Therefore, biodegradable antigen-loaded MP represent a valuable system for the delivery of antigen to APC. Recently, Waeckerle-Men and Groettrup [25] reported on the ex vivo loading of autologous DC with antigen-containing MP in view of designing cellular vaccines for tumor immunotherapy. Importantly, the loading of immature DC with antigenfree PLGA MP exerted no negative effect on the pivotal properties of the DC, as assessed in vitro [26]; indeed MPloaded immature DC preserved their capacity to mature upon appropriate stimulation, as assessed by the upregulation of the cell surface markers CD80, CD83, and CD86. Furthermore, the MP-loaded DC also maintained the functional property to secrete immunostimulatory cytokines such as IL-12 and TNF- $\alpha$, which promote $\mathrm{T}_{\mathrm{h} 1}$ and CTL responses [22,26,27].

The use of polymeric antigen delivery systems requires the consideration of potential immunomodulating activities of synthetic polymers [28]. Both synthetic [27,29] and natural $[30,31]$ polymers as well as their corresponding monomers [32] may exhibit significant immunomodulating activity, although little is known about the underlying mechanisms. Suggested mechanisms include the interaction of the polymers with distinct surface receptors, such as the binding of polysaccharides to the mannose receptor [33], or physicochemical mechanisms, e.g., the acidification of endosomes leading to MHC class-II presentation of antigens upon polymer degradation [14]. Cationic polymers, such as chitosan, poly(ethylene imine) (PEI) and protamine, may possibly exert sufficient buffering capacity for the acidic endosomes to induce cytoplasmic antigen delivery for antigen presentation via the MHC class-I molecule [28,34]. Thus, polymers and derived formulations may have great impact on the elicitation of an immune response. Taking into account other parameters like antigen dose and release kinetics, the complexity of antigen delivery systems becomes obvious.

In this study, we prepared and concomitantly coated PLGA MP with the cationic aminodextran, chitosan, PEI, poly(L-lysine) (PLL), and protamine, by means of a modified solvent extraction process [17]. The differently coated PLGA MP were then tested in vitro in the context of immature and matured DC to elucidate their effect on crucial cellular functions such as phagocytosis, phenotype, migration capacity, cytokine secretion, and viability. The rationale of this study was to (i) obtain PLGA MP with a polycationic and chemically reactive surface for future surface binding of nucleic acid vaccines and/or immunostimulatory molecules; (ii) increase our knowledge on the suitability of various polycationic materials for PLGA MP coating; and (iii) ascertain that the coating material does not alter crucial functional properties of DC upon PLGA particle uptake. The general absence of any immunobiological effects by the particulate carrier materials on the DC was indeed considered to be essential in view of controlling immune responses by additional immunostimulants or eventually utilizing these particles for ex vivo loading of monocyte-derived DC (MoDC) for use as cellular vaccines.

\section{Materials and methods}

\subsection{Materials}

PLGA (50:50) of $35 \mathrm{kDa}$ with uncapped end-groups (Resomer RG503 H) was from Boehringer-Ingelheim (Ingelheim, Germany). Coumarin-6, salmine sulfate derived from salmon sperm (protamine sulfate with four arginine residues at the C-terminus), PEI solution $(\sim 50 \%)$, and PLL hydrobromide of $70-150 \mathrm{kDa}$ were from Fluka/ Sigma (Buchs, Switzerland). The $40 \mathrm{kDa}$ aminodextran used was from Molecular Probes (Leiden, The Netherlands). Chitosan (Seacure ${ }^{\circledR}$ CL110) was kindly offered by Pronova Biopolymer (Drammen, Norway). 
Pharmaceutical-grade chitosan $\left(\mathrm{Chitech}^{\circledR} 00 \mathrm{M}-010\right)$ was purchased from Carmeda AB (Upplands Väsby, Sweden). AIM V medium used for in vitro DC culture was purchased from Invitrogen (Groningen, The Netherlands), Ficoll-Paque ${ }^{\circledR}$ from Pharmacia (Uppsala, Sweden), and the human recombinant cytokines IL-4, TNF- $\alpha$, IL-1 $\beta$, and IL-6 from CellGenix (Freiburg, Germany). Human GM-CSF (Leukine ${ }^{\circledR}$ ) was obtained from Immunex (Seattle, WA, USA), prostaglandin E2 (PGE-2, Prostin E2) from Pharmacia \& Upjohn (Dübendorf, Switzerland), and human chemokine CCL21 from R\&D Systems (Wiesbaden-Nordenstadt, Germany).

\subsection{Preparation of $M P$}

MP were made by microextrusion-based solvent extraction using a static multilamination-type micromixer, as previously described [35], with slight modifications. PLGA was dissolved at $5 \%(\mathrm{w} / \mathrm{w})$ in DCM as organic phase. Fluorescent MP were prepared by adding $0.5 \mu \mathrm{g}$ Coumarin- 6 per mg PLGA to the organic phase. MP coated with polyelectrolyte polymers were obtained by dissolving the coating polymer in the aqueous extraction fluid: PEI, PLL, and protamine sulfate were dissolved in pure water, both chitosan types and aminodextran were dissolved in $0.1 \mathrm{~N} \mathrm{HCl}$. The polyelectrolyte concentration in the extraction fluid was $0.5 \%(\mathrm{w} / \mathrm{w})$ for both chitosan types, PEI and protamine sulfate, and $0.1 \%(\mathrm{w} / \mathrm{w})$ for PLL and aminodextran. For "uncoated" PLGA MP, the solvent extraction and particle collection fluid was a $0.5 \%(\mathrm{w} / \mathrm{w})$ solution of PVA. All coated PLGA MP formulations were collected in purified water. After extrusion in the micromixer, the dispersion containing the MP was transferred into a flat open bowl, gently stirred and kept in a laminar air hood for $30 \mathrm{~min}$ for solvent removal and hardening of the particles. The particles were collected on a $0.2 \mu \mathrm{m}$ pore-size mixed cellulose ester membrane filter (Schleicher \& Schuell, Dassel, Germany) and dried at $20 \mathrm{mbar}$ and room temperature for $24 \mathrm{~h}$.

\subsection{Zeta potential measurement}

Surface charge of the MP was determined by zeta potential measurement (Malvern Zetasizer 3000 HSA, Malvern, UK). Approx. $1 \mathrm{mg}$ of MP was suspended in $10 \mathrm{ml}$ of $1 \mathrm{~mm} \mathrm{KCl}(\mathrm{pH}=7.66)$. The $\mathrm{pH}$ value of the medium after particle dispersion was measured and found to be in the range of 7.94 $\pm 0.06-8.17 \pm 0.06$. The minor effect of the different particles on the $\mathrm{pH}$ of the dispersion media were neglected. Mean zeta potential values were calculated from five measurements per sample.

\subsection{Generation of $M o D C$}

Immature MoDC were generated from human peripheral blood mononuclear cells (PBMC), as previously described $[36,37]$. In brief, PBMC were separated on Ficoll-Paque and resuspended at $6 \times 10^{6} \mathrm{cells} / \mathrm{ml}$ in AIM V medium. The cells were seeded into tissue culture flasks (TPP, Trasadingen, Switzerland) to let them adhere on the plastic surface for $2 \mathrm{~h}$ at $37^{\circ} \mathrm{C}$. Nonadherent cells were removed by rinsing three times with PBS. Tightly adhering monocytes were further cultured in DC medium (AIM V medium containing $1000 \mathrm{U} / \mathrm{ml}$ of GM-CSF and $800 \mathrm{U} / \mathrm{ml}$ of IL-4) in the absence of fetal calf serum. On day 5 or 6 , non-adherent or loosely adherent cells were harvested. The immature MoDC were tested for the presence (positive sign) or absence (negative sign) of the following surface markers: $\mathrm{CD} 11 \mathrm{c}^{+}$, $\mathrm{CD} 14^{-}, \mathrm{CD} 16^{-}, \mathrm{CD} 19$ (and $\left.\mathrm{CD} 20\right)^{-}, \mathrm{CD} 40^{+}, \mathrm{CD} 80^{+}, \mathrm{CD} 83^{\text {low }}$ or neg, $\mathrm{CD}^{+} 6^{+}, \mathrm{CCR}^{-}$, and $\mathrm{MHC}$ class $\mathrm{I}^{+}$and $\mathrm{II}^{+}[26,38]$. For MoDC maturation, immature MoDC were stimulated with a proinflammatory cytokine/PGE-2 cocktail (20 ng/ml TNF- $\alpha, 10 \mathrm{ng} / \mathrm{ml}$ IL-1 $\beta, 1000 \mathrm{U} / \mathrm{ml}$ IL-6, and $1 \mu \mathrm{g} / \mathrm{ml}$ PGE-2 dissolved in fresh DC medium) for additional $48 \mathrm{~h}$.

\subsection{Phagocytosis of $M P$ by $M o D C$}

Fluorescent MP (Table 1) were dispersed in DC culture medium and incubated with immature MoDC at $37^{\circ} \mathrm{C}$ overnight in 24-well cell culture plates (Cellstar, Greiner Bio-One, Frickenhausen, Germany); the concentration of MP was $0.2 \mathrm{mg}$ per $10^{6}$ cells. The used amount of MP corresponded to a total particle surface area of $3.7 \mathrm{~cm}^{2}$ per $10^{6}$ cells. Phagocytosis of the particles was assessed by FACS analysis. Samples containing DC were resuspended in PBS containing $2 \% \mathrm{FCS}$ and analyzed on a FACScan ${ }^{\circledR}$ flow cytometer (Becton Dickinson, Basel, Switzerland).

\subsection{MoDC phenotype analysis by FACS analysis}

The phenotype of immature and mature MoDC in absence or presence of MP was analyzed in a consecutive experiment. Firstly, the MP were incubated with immature moDC for $20 \mathrm{~h}$ at $37^{\circ} \mathrm{C}$, as described in Section 2.5. Control cells without MP were treated identically. Then, half of the immature MoDC that carried MP and of control cells were analyzed for phenotype, whereas the other half of the cells were further stimulated by the maturation cocktail (for details on DC maturation see Section 2.4) prior to phenotype analysis. Phenotype analysis of MoDC was made on a FACScan ${ }^{\circledR}$ flow cytometer (Becton Dickinson) after staining with the following $\mathrm{mAbs}$, according to the manufacturers' protocols: FITC-labeled anti-CD83 (Immunotech, Berlin, Germany), FITC-labeled anti-CD86, PE-labeled anti-CD80, and FITC-labeled anti-HLA-DR (all from Pharmingen, Basel, Switzerland). The respective isotype controls were FITC-labeled mouse IgG1, IgG2a, and IgG2b (Pharmingen). Rat antihuman CCR7 mAb [39] was kindly provided by Dr. Reinhold Förster (University Erlangen-Nürnberg, Germany). FITC-conjugated goat-antirat IgG (Jackson Immunoresearch, F. Hofmann-La Roche, Basle, Switzerland) was used as secondary antibody for anti-CCR7 staining.

\subsection{MoDC migration assay}

In vitro migration of MoDC was measured by a chemotaxis assay, as previously described [37]. Briefly, $600 \mu \mathrm{l}$ of DC medium, with or without $250 \mathrm{ng} / \mathrm{ml} \mathrm{CCL} 21$, was added to the bottom chamber of a 24-well Transwell plate with polycarbonate filters of $5 \mu \mathrm{m}$ pore size (Corning Costar, Cambridge, MA), while $10^{5} \mathrm{MoDC}$, obtained as described above,

Table 1

PLGA MP formulations tested for their effect on MoDC phenotype and maturation, migration capacity, cytokine release and viability. For determination of MoDC phagocytosis, MP were fluorescently labeled with Coumarin 6

\begin{tabular}{llll}
\hline Label & Coating polymer & $\begin{array}{l}\text { Coating polymer concentration } \\
\text { in extraction fluid \% }(\mathrm{w} / \mathrm{w})\end{array}$ & Collection fluid \\
[solvent] & $0.5[\mathrm{water}]$ & $0.5 \%$ PVA \\
\hline PLGA & PVA & $0.1[0.1 \mathrm{~N} \mathrm{HCl}]$ & Water \\
AmDex & Aminodextran & $0.5[0.1 \mathrm{~N} \mathrm{HCl}]$ & Water \\
ultrapureChit & Chitosan Chitech & $0.5[0.1 \mathrm{~N} \mathrm{HCl}]$ & Water \\
Chit & Chitosan CL 110 & $0.5[$ water] & Water \\
PEI & Poly(ethylene imine $)$ & $0.1[$ water] & Water \\
PLL & Poly(L-lysine) & $0.5[$ water] & Water \\
Protamine & Protamine sulfate & & \\
\hline
\end{tabular}


were dispersed in a total volume of $100 \mu \mathrm{LC}$ medium and added to the upper chamber. The Transwell plates were then incubated at $37^{\circ} \mathrm{C}$ for $3 \mathrm{~h}$. After careful removal of the upper chamber, a $500 \mu \mathrm{l}$ aliquot of medium containing migrated cells were collected from the bottom chamber and analyzed by FACS (counting for $60 \mathrm{~s}$ ) using CellQuest software (Becton Dickinson). Each experiment was performed in duplicate. The migration ratio was calculated as number of migrated MoDC carrying MP divided by the number of migrated MoDC free of MP.

\subsection{Cell viability and apoptosis assay}

Cell viability was determined by Annexin V staining, which detects apoptotic cells; the assay was conducted according to the manufacturer's protocol (Pharmingen). Cells were labeled with Annexin V-FITC and propidium iodide (PI) to identify apoptotic cells by FACS analysis; live cells remained Annexin V-and PI-negative.

\subsection{Cytokine ELISA}

Cytokine secretion of immature and mature MoDC, in the absence or presence of MP, was analyzed in a consecutive experiment, as described in Section 2.6. The supernatants of immature and mature MoDC, stimulated under the indicated experimental conditions in the presence or absence of MP, were collected and analyzed for cytokine excretion using commercially available cytokine ELISA kits for IL-10 (Pharmingen) and IL-12p70 (Endogen, Woburn, MA); the assays were conducted according to the manufacturers' protocols.

\subsection{Statistical analysis}

Samples were evaluated using ANOVA where appropriate. If indicated, ANOVA was followed by a post-hoc assessment using the Tukey HSD method. Differences were considered significant when $p$-values were equal or less than 0.05 .

\section{Results}

\subsection{Zeta potential of the $M P$}

The zeta potential differed significantly between uncoated and differently coated PLGA MP (Fig. 1).

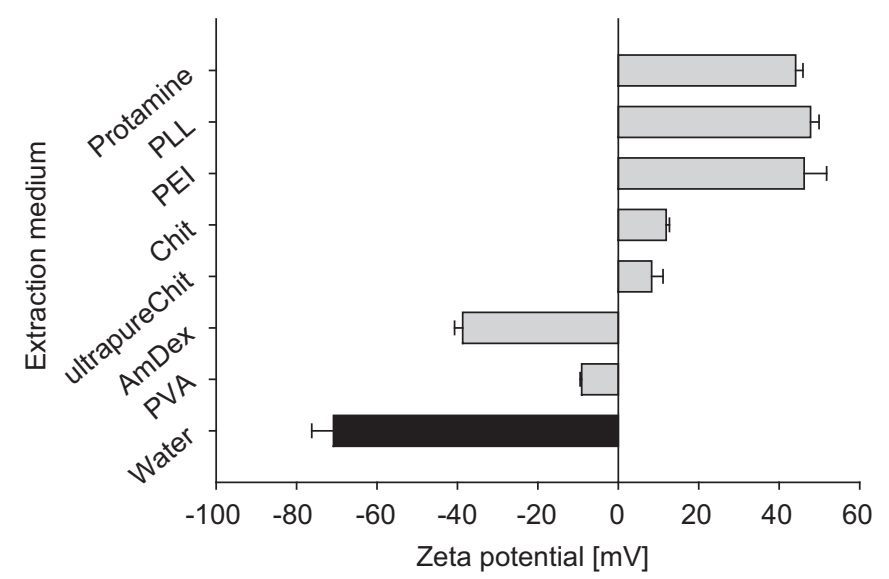

Fig. 1. Zeta potential $[\mathrm{mV}]$ of uncoated and polycation-coated PLGA MP. The "uncoated" MP were prepared with the help of water (water) or $0.5 \%$ PVA solution as solvent extraction medium; the coated MP were prepared with the help of aqueous solutions of the various polycations as solvent extraction phase. Further details on the composition of the applied solvent extraction and collection media are given in Table 1.
Uncoated PLGA MP, prepared either in pure water or in $0.5 \%$ aqueous PVA as solvent extraction and MP collection fluids, served as reference particles. The zeta potential of the PLGA MP prepared in pure water was approx. $-70 \mathrm{mV}$. The use of $0.5 \%$ PVA as extraction/ collection fluid shifted the zeta potential of the PLGA MP to $-10 \mathrm{mV}$. Aminodextran-coated PLGA MP exhibited a zeta potential of approx. $-40 \mathrm{mV}$. Conversely, the zeta potential was strongly positive when the PLGA MP were coated with protamine, PLL, and PEI $(+44$ to $+48 \mathrm{mV})$, or slightly positive $(+8$ to $+12 \mathrm{mV})$ when chitosan was used for coating.

\subsection{Phagocytosis of PLGA MP by MoDC and cell viability}

All fluorescently labeled MP formulations were efficiently taken up by immature MoDC. Generally, uptake efficiency was in the range of $50-60 \%$ and did not differ with the coating polymer. This data agree well with previous one on the uptake of uncoated PLGA MP $[25,26]$.

The viability of MoDC ranged from $67 \%$ to $73 \%$ and was not adversely affected by the phagocytosis of the different uncoated and coated particle types. This data also agree well with previous one of uncoated PLGA MP [26].

\subsection{Effect of MP uptake on MoDC phenotype and maturation}

The DC surface markers CD80, CD83, CD86, and HLA-DR represent important surface molecules for antigen presentation and T-cell activation [22]. By means of FACS analysis, we addressed the question whether the phagocytotic uptake of uncoated or differently coated PLGA MP by MoDC had a significant impact on the expression of the surface markers and DC maturation. Interestingly, immature MoDC exhibited similar expression levels of CD80, CD83, CD86, and HLA-DR irrespective of MP phagocytosis (Fig. 2, first three columns). Fig. 2 illustrates the full set of data for chitosan-coated PLGA MP, which are representative for all the other PLGA MP formulations. Expression levels were low for CD80 and CD83, but high for CD86 and HLA-DR. The absence of significant differences between differently coated MP formulations is shown in Fig. 4 (black columns). Importantly, the addition of the proinflammatory cytokine/PGE-2 cocktail to the immature MoDC that had ingested MP still afforded DC maturation as evidenced by the up-regulation of CD80, CD83, CD86, and HLA-DR (Fig. 2, last three columns). Again, the expression levels were similar irrespective of MP phagocytosis.

The transition of MoDC from their antigen-capturing to an antigen-presenting state was further confirmed by the down-regulation of the macrophage mannose receptor (MMR) upon cytokine/PGE-2-induced maturation. Immature DC (Fig. 3A) exhibited high expression levels of MMR, both before and after uptake of uncoated or 

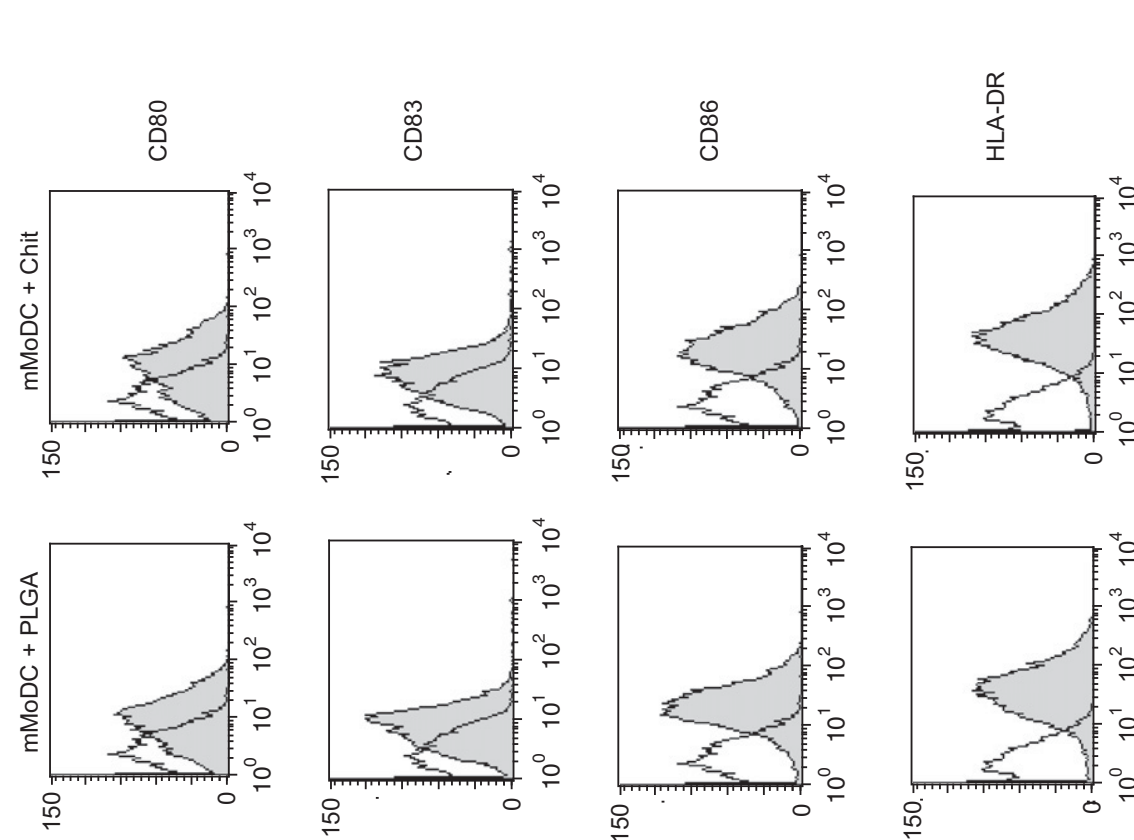

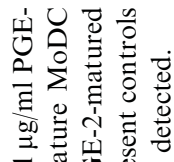

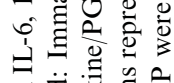

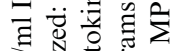

응

응

今.

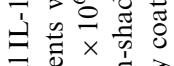

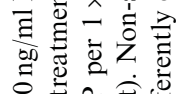

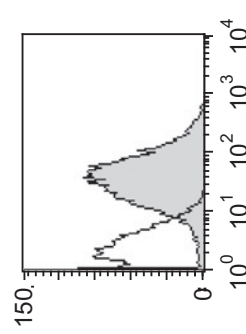

今 $\sum_{00}$

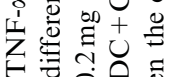

ㄹㅇ웡

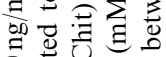

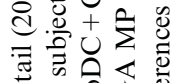

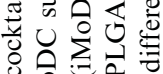
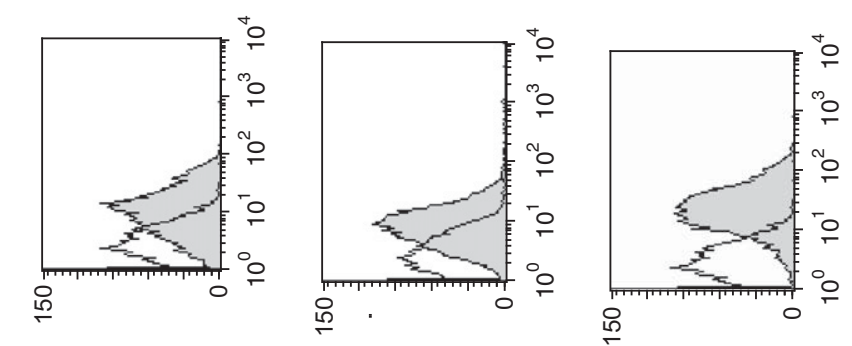

초

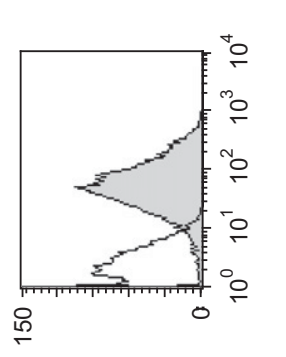

넝

严郘

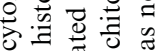

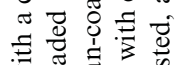

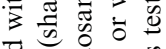

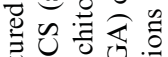

要迲专远
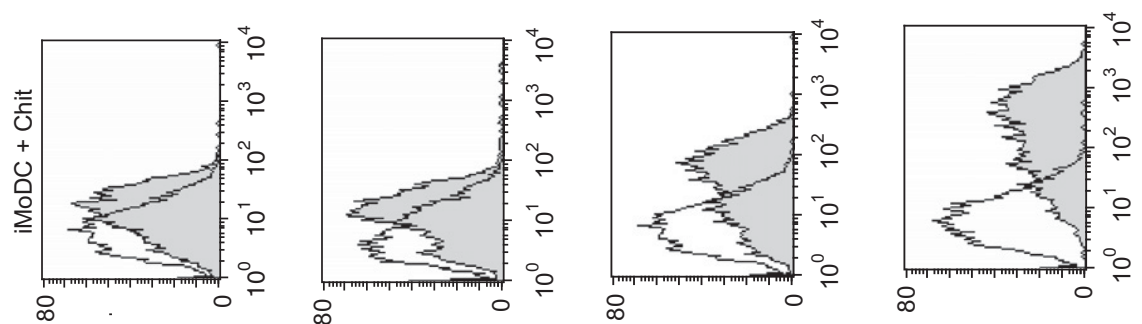

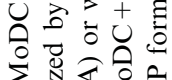

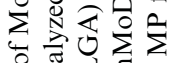

व

号志氙

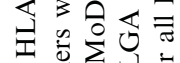

현

๘
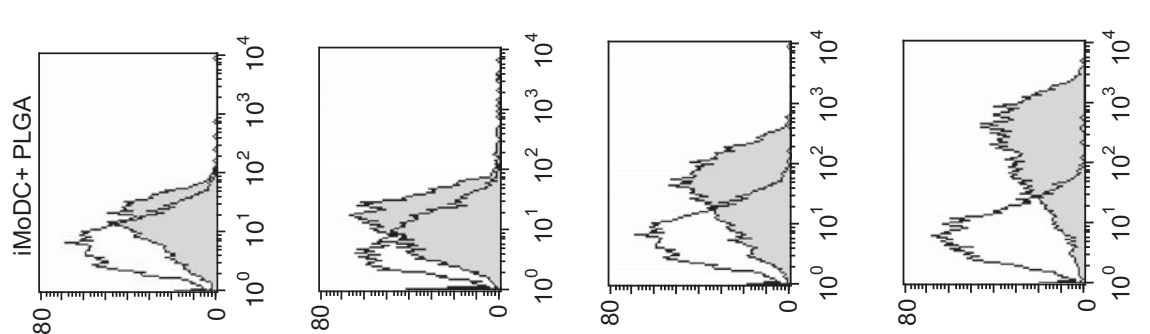

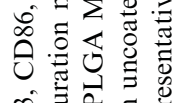

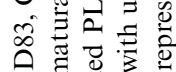

O

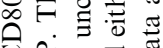

已

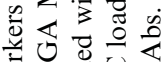

元
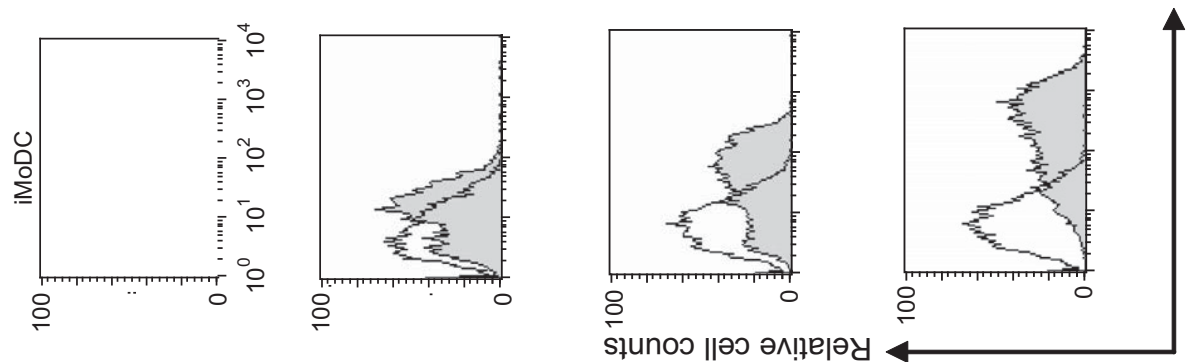

它额

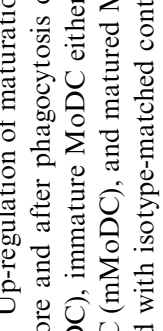

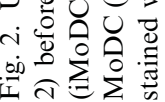




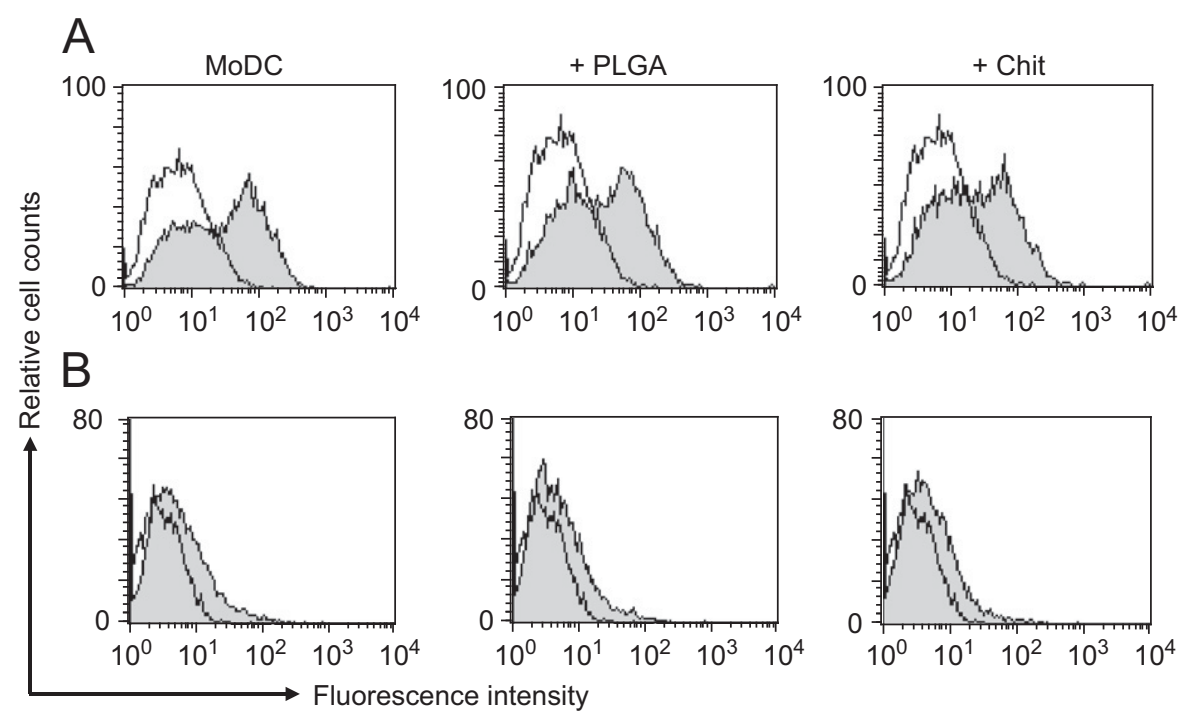

Fig. 3. Down-regulation of the macrophage mannose receptor (MMR) upon cytokine-induced maturation of DC (see Section 2). Panel A: High expression of MMR by immature DC. Panel B: Low expression of MMR by mature DC. Data on MoDC with ingested uncoated PLGA MP (+ PLGA) and ingested chitosan-coated PLGA MP (+ Chit) are representative for all the other PLGA MP formulations.

chitosan-coated PLGA MP. Mature DC (Fig. 3B) showed low MMR expression, again before and after MP uptake.

For testing the statistical significance of up- and downregulation of CD80, CD83, CD86, HLA-DR, and MMR on immature and matured MoDC, we pooled the FACS analysis data $(n=8)$ of the cells incubated or not with the differently coated PLGA MP (MoDC alone, MoDC incubated with uncoated PLGA MP and with AmDex-, ultrapureChit-, Chit-, PEI-, PLL-, and protamine-coated MP); the mean percentage of cells that were positive for the different maturation markers was compared (Fig. 4A). For each surface marker, the percentage of pooled positive cells varies very little (small error bars) for both the immature and matured MoDC indicating that the MP phagocytosis did not exert any effect on the measured phenotypical characteristics of the MoDC. Importantly, the MoDC maturation by a maturation cocktail was not hampered by the presence of previously ingested MP. The consistent behavior of the immature and matured MoDC towards the different MP formulations is further illustrated by the percentage of CD83- and CD86-positive cells that had been incubated with the different MP formulations (Fig. 4B and C). The data revealed no major differences for the different MP formulations. Comparable data were also obtained for CD80-, HLA-DR- and MMR-positive cells (data not shown).

\subsection{Migration capacity of MoDC in vitro before and after phagocytosis of PLGA MP}

A crucial attribute of DC is their capacity to migrate from peripheral tissues to the lymph nodes and elicit an immune response by priming $\mathrm{T}$ lymphocytes [40]. This migration is induced by chemokine receptor ligands (CCL), e.g., CCL21, which bind to chemokine receptors (CCR) expressed on mature MoDC, e.g., CCR7 [41]. By using a Transwell chemotaxis assay, we addressed the question, whether the migration of mature DC towards CCL21 is impaired by the presence of ingested PLGA MP formulations (Fig. 5). First, the control assay revealed a significant difference between DC migration towards control medium and medium containing CCL21 (Fig. 5A). The migration capacity of MoDC was expressed as migration ratio, i.e., the percentage of migrated MoDC carrying MP over the percentage of migrated MoDC without ingested MP, which was set to 1.0 (Fig. 5B). The data reveal only minor differences in MoDC migration as a function of the presence and type of ingested MP.

The data derived from the migration assay also agrees with the surface expression of CCR7 on mature DC (Fig. 6). The expression level was similar for the control MoDC (MoDC alone) and the DC that had phagocytosed uncoated PLGA MP (Fig. 6B), chitosan-coated PLGA MP (Fig. 6C), or any of the other coated MP formulations (data not shown). Incidentally, the expression of CCR7 on immature DC is not shown, because previous work has already demonstrated that CCR7 is not expressed on immature DC [37].

\subsection{Cytokine secretion of MoDC after phagocytosis of MP}

Beside the expression of maturation markers, secretion of cytokines by mature MoDC is crucial for T-cell activation and efficient antigen presentation. By measuring cytokine secretion of MoDC prior to and after maturation and with or without ingested PLGA MP, we addressed the question whether the previously ingested MP exerts an influence on cytokine secretion of matured MoDC in vitro.

MoDC treated with the maturation cocktail secreted markedly increased levels of IL-10 and IL-12p70, 
irrespective of the presence or absence of ingested MP (Fig. 7A and B). Mature MoDC that had previously phagocytosed uncoated PLGA MP showed highest IL-10

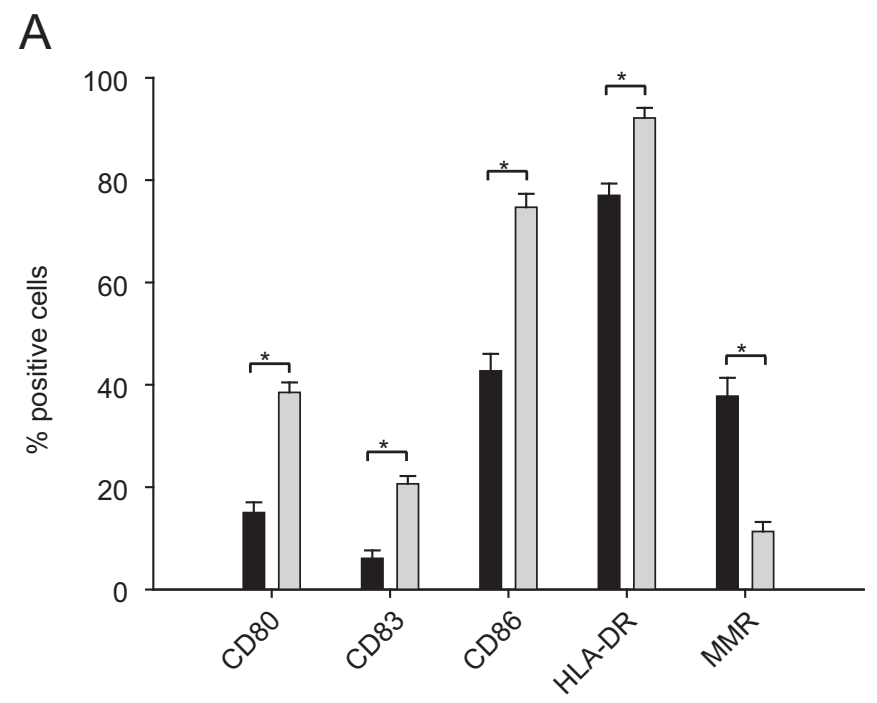

B

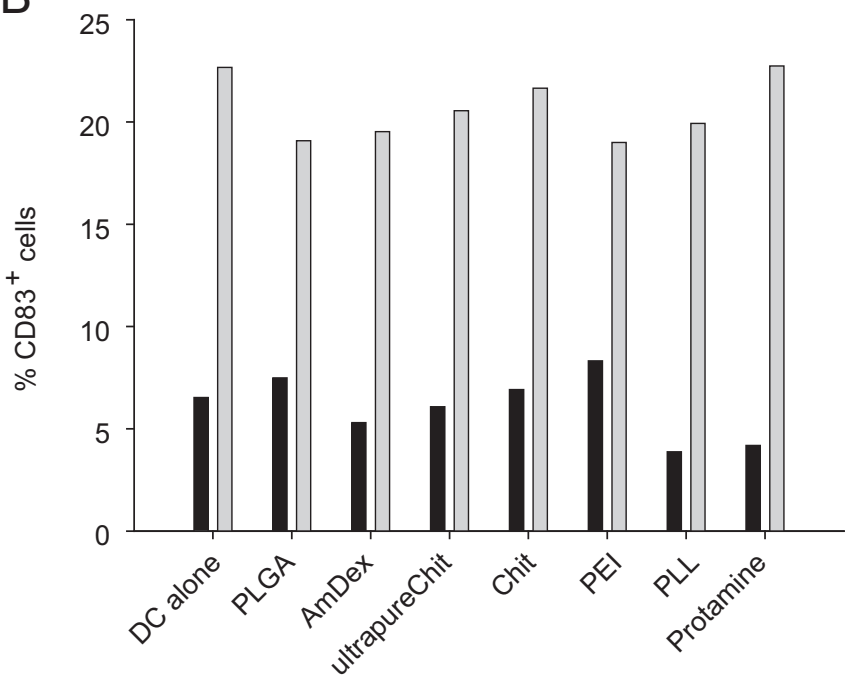

C

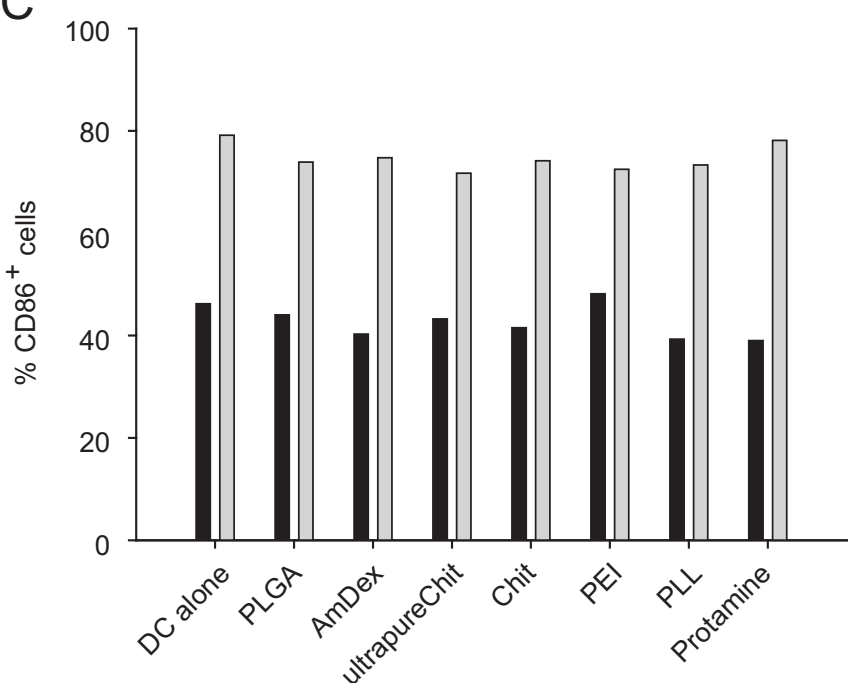

secretion (Fig. 7A), whereas the MoDC that had ingested the different coated MP formulations secreted IL-10 at similar amounts as did the control cells, which had not been incubated with MP. Interestingly, immature MoDC secreted the highest IL-10 amounts after phagocytosis of PEI-coated PLGA MP (Fig. 7A). In addition, mature MoDC that had phagocytosed the PEI-coated PLGA MP secreted also the highest level of IL-12p70 (27 pg/ml), which was approximately two-fold above the secretion level of the control cells (DC alone) and of the MoDC that contained uncoated PLGA MP (13 and $14 \mathrm{pg} / \mathrm{ml}$, respectively) (Fig. 7B). All other IL-12p70 levels were in the range of the quantitation limit $(8 \mathrm{pg} / \mathrm{ml})$. Altogether, phagocytosis of the various PLGA MP formulations did not greatly influence the cytokine secretion pattern of immature and mature MoDC in vitro, except for PEI-coated PLGA MP.

\section{Discussion}

Various types of microparticulate carriers have been developed and explored for their use as systems for antigen delivery $[28,42,43]$. The benefits of biodegradable MP in vaccination and their immuno-adjuvant property have been well documented. Especially PLGA MP have been widely described $[2,20,43]$. In addition to their potential for standard immunization, e.g., through subcutaneous administration, PLGA MP have also been proposed as a promising delivery system for the ex vivo antigen loading of MoDC for use as cellular vaccine [25]. The current study aimed at clarifying the question whether polyelectrolytecoated PLGA MP may be used for these purposes. In particular, we examined the effect of polyelectrolyte coatings on PLGA MP on the phagocytotic particle uptake by MoDC, and on the phenotype and functionality of immature and mature MoDC in vitro. The tested MP types included PLGA MP left uncoated and coated with cationic aminodextran, chitosan, PEI, PLL, and protamine. Our interest to explore such cationic surface coatings was motivated by their usefulness for future chemical conjugation of bioactive ligands or physical attachment of nucleic acid therapeutics.

Fig. 4. Effect of different MP formulations on DC surface marker expression. Panel A: Statistical analysis of surface marker expression (CD80, CD83, CD86, HLA-DR, and MMR) upon maturation of immature MoDC (black columns) to mature MoDC (gray columns) by a proinflammatory cytokine/PGE-2 cocktail. MoDC maturation was induced after loading of the cells with different MP formulations. Data represent the mean percentage of positive cells \pm SD under $n=8$ different conditions, namely MoDC without MP as control, and upon phagocytosis of uncoated PLGA MP, and of PLGA MP coated with aminodextran (AmDex), ultrapure Chitosan (ultrapureChit chitosan (Chit)), poly(ethylene imine) (PEI), poly(L-lysine) (PLL), and protamine, respectively. The significance of up- or downregulation within each marker group was proven by ANOVA $\left({ }^{*} p<0.05\right)$. No significant difference between MP formulations was observed. Panels $B$ and $C$ : Illustration of the lack of influence of different PLGA MP coatings on the up-regulation of CD83 and CD86 upon cytokine/PGE-2-induced maturation of MoDC that had previously been loaded with the different MP types. 
A

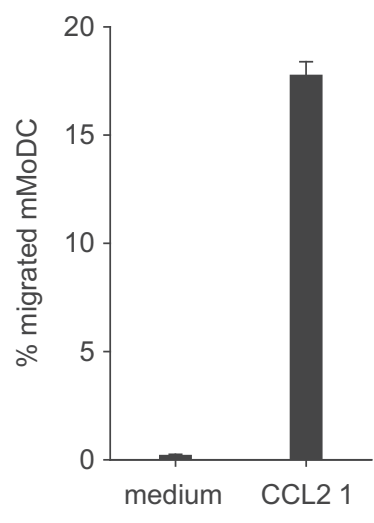

B

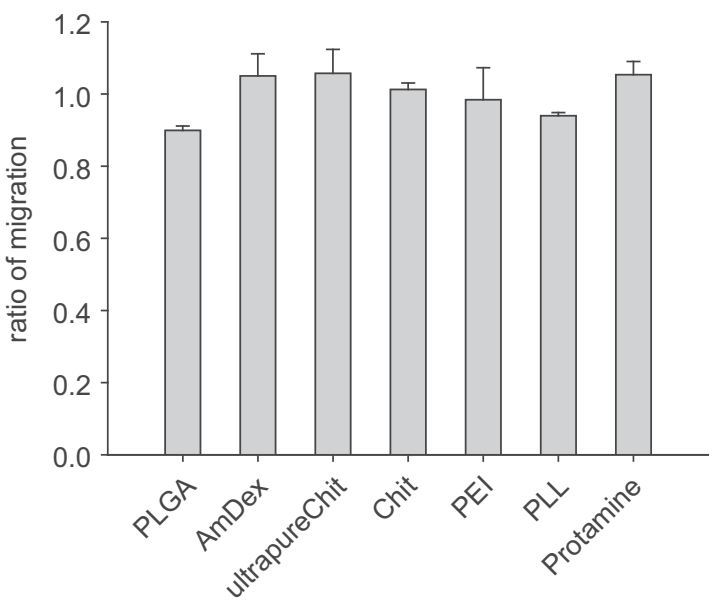

Fig. 5. Influence of ingested PLGA MP on the migration capacity of MoDC towards CCL21. Immature MoDC were first loaded with different coated PLGA MP types and then matured with a cocktail containing TNF- $\alpha$, IL-1 $\beta$, IL-6, and PGE-2 (see Section 2). Panel A: Control experiment showing the difference between CCL21-induced chemotaxis of MoDC and spontaneous cell migration towards the cell culture medium without chemokines. The data reveal a significantly higher migration of MoDC towards CCL21 than towards chemokine-free medium. Panel B: Migration capacity of mature MoDC loaded with phagocytosed MP relative to MoDC alone. The migration ratio was calculated as: number of migrated MoDC loaded with MP/number of migrated MoDC not loaded with MP. The data are expressed as the mean of duplicate measurements \pm SEM. The uptake of MP did not impair the migration capacity of MoDC irrespective of MP uptake and type of ingested formulation.
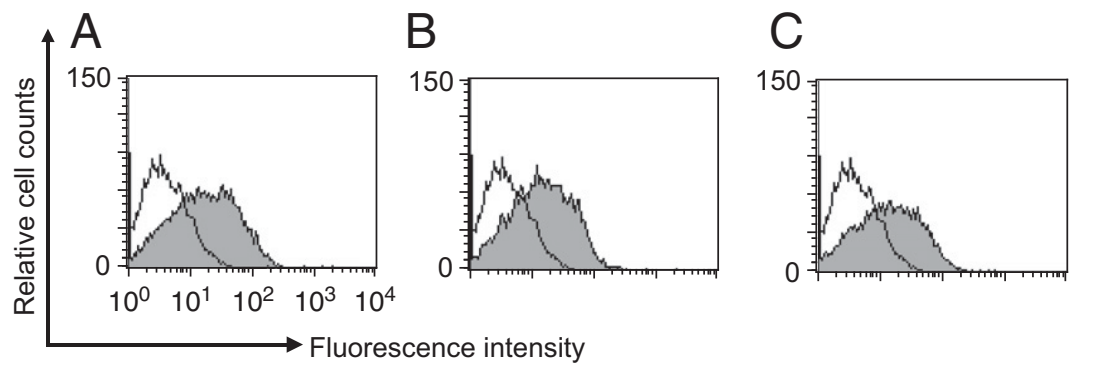

Fig. 6. Expression of chemokine receptor CCR7 (shaded area) on mature MoDC. Non-shaded histograms represent control cells stained with secondary antibody. For maturation, MoDC were treated with the maturation cocktail, either in the absence of MP (panel A), or after uptake of PLGA MP (panel B) or of chitosan-coated PLGA MP (panel C). The uptake of MP did not impair the expression of CCR7. PLGA MP (panel B) and chitosan coated PLGA MP (panel C) are representative for all other MP formulations, which revealed similar results (data not shown).

While all the PLGA MP formulations studied exhibited comparable size distributions $\left(d_{10 \%}-d_{90 \%}\right.$ of $\left.1-15 \mu \mathrm{m}\right)$, they differed significantly in their zeta potential (Fig. 1). Uncoated PLGA MP prepared in the absence or presence of PVA in the solvent extraction fluid possessed a highly negative $(-70 \mathrm{mV})$ or slightly negative $(-10 \mathrm{mV})$ zeta potential, respectively. Coating with aminodextran resulted in an intermediate negative potential $(-40 \mathrm{mV})$, whereas coating with chitosan $(+15 \mathrm{mV})$ or with protamine, PLL, or PEI $(>+40 \mathrm{mV})$ shifted the potential to positive values.

Irrespective of the used polyelectrolyte and surface charge, all MP formulations were efficiently phagocytosed by immature MoDC. When the immature MoDC, carrying or not carrying MP, were matured with the help of a cytokine cocktail, the cell surface markers CD80, CD83, and CD86 were up-regulated, the mannose receptor was down-regulated, and cytokine secretion was increased.
These phenotypic changes reflect the transition from an antigen-capturing to an antigen-presenting state of the DC [22]. Such DC maturation is naturally induced by pathogens and pathogen-associated molecules, but can be impaired by certain drugs [44]. For this reason, we wanted to examine whether the presence of phagocytosed PLGA MP carrying various surface coatings would influence the phenotype of immature and matured DC. The presented data (Figs. 2-4) confirmed previous ones [20] showing that uncoated PLGA MP did not impair the phenotype and crucial functional properties of MoDC in vitro. In the present study, none of the differently coated PLGA MP impaired the MoDC maturation induced by a cytokine/ PGE-2 cocktail; CD80, CD83, CD86, and HLA-DR were up-regulated, and the MMR down-regulated to a comparable extent, irrespective of the presence and type of previously ingested MP. The data emphasize that none of 


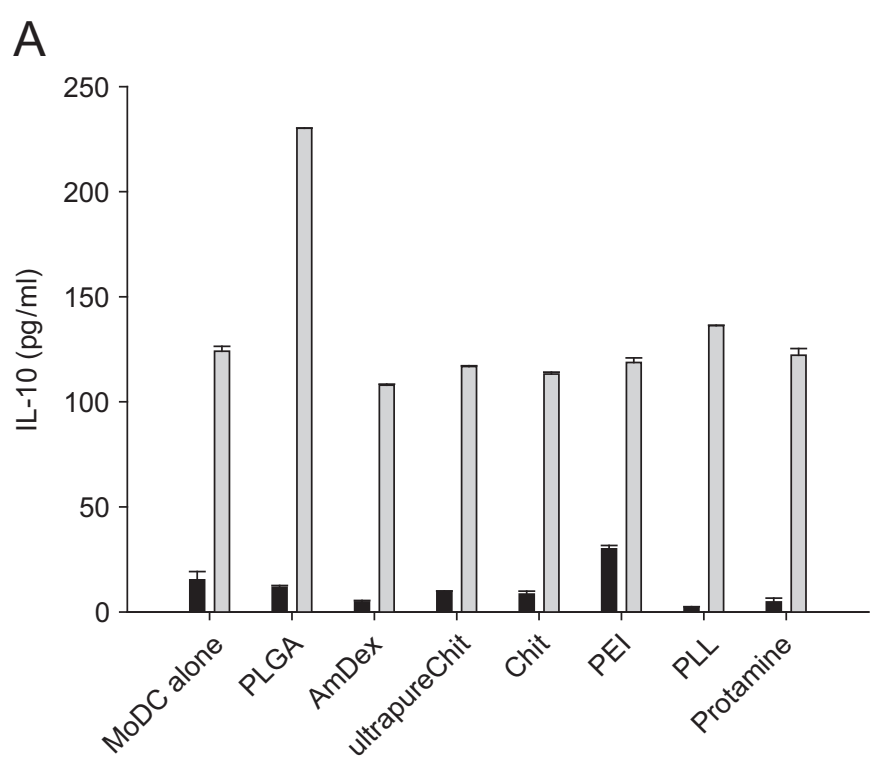

B

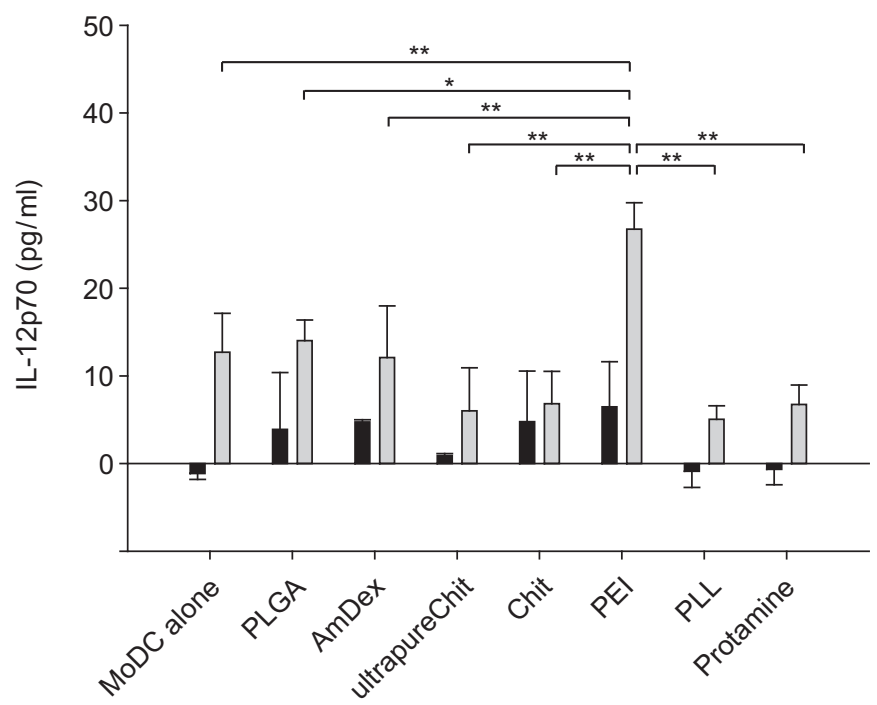

Fig. 7. Cytokine secretion of MoDC prior to or after maturation (for details see Section 2) before (MoDC alone) and after loading with different MP formulations (for details see Table 1). Supernatants of $1 \times 10^{6}$ of immature MoDC (black columns) or mature MoDC (gray columns) were collected and analyzed for the cytokines IL-10 (panel A) and IL-12 (panel $B$ ) using ELISA. Quantitation limits were $8 \mathrm{pg} / \mathrm{ml}$ for IL12 and $5 \mathrm{pg} / \mathrm{ml}$ for IL-10. The data represent the means of two (panel $A$ ) or four (panel $B$ ) independent experiments \pm SD. For IL-12, statistical differences were proven by ANOVA followed by a Tukey HSD posthoc test $(* p<0.05$ and $* * p<0.01)$.

the tested PLGA MP formulations impaired the cytokine/ PGE-2-induced maturation of DC [22]. The excellent compatibility between PLGA MP and MoDC was further supported by a chemotaxis assay, showing that MPcarrying matured DC migrated as efficiently towards CCL21 as did particle-free matured DC (Fig. 5). Furthermore, matured DC revealed high expression levels of CCR7 irrespective of the presence and type of ingested MP (Fig. 6). These findings are in agreement with recent data on the preservation of phenotypic and functional properties of DC after phagocytosis of PLGA MP in vitro [26]. In the latter study, the compatibility between particles and DC was considered highly beneficial in view of using such particles to load MoDC with antigen and exploit antigenloaded DC as cellular vaccines [20].

Similar to this data on surface markers, data on cytokine secretion of matured DC that carried different PLGA MP types did not generally reveal any undesired effect of the phagocytosed PLGA MP in vitro. With the exception of PEI-coated PLGA MP, polyelectrolyte-coated PLGA MP did not affect the cytokine secretion of immature and mature MoDC in vitro (Fig. 7). PLGA MP coated with PEI induced high levels of IL-12p70, which is commonly recognized as a marker for Th1-oriented immune responses $[45,46]$. Although the potential of PEI as a tool for gene delivery has been described, only little is known about its putative capacity to stimulate systemic immune responses [47]. Further investigations are therefore needed to assess the capacity of PEI-coated PLGA MP to influence the type and extent of the immune response.

Although costimulatory markers and cytokine profiles provide a reasonable appreciation of the functional status of DC, mature DC must also be capable to stimulate lymphocytes. In a previous work on similar MP, we have demonstrated that immature MoDC, with and without ingested PLGA MP, were poor T-cell stimulators [26]. After maturation with lipopolysaccharide (LPS), the MoDC, with and without ingested PLGA MP, induced strong T-cell proliferation.

Different reports have addressed the immunobiological role of unmodified PLGA MP. In a previous in vitro study we have shown that uncoated PLGA MP, which had been prepared by spray-drying, do not induce maturation of MoDC in vitro [26]. Thus, the main immunobiological function of PLGA MP is indeed the passive targeting of entrapped antigens into APC [48]. The lack of DC maturation upon PLGA MP exposure was recently confirmed by Wischke et al. [49], who used both anionic and cationic PLGA MP coated with CTAB, chitosan, or DEAE-dextran. Other studies reported on a slight MoDC stimulation upon exposure to PLGA MP, which was more pronounced when bacterial DNA was admixed to the particles [24]. A rather intriguing result has been presented by Yoshida and Babensee [27], who recently reported on an enhanced maturation of MoDC upon contact with either PLGA MP or PLGA films. This maturation was demonstrated by an increased expression of CD40, CD80, and CD86. The latter data have been further supported by a study by Babensee and Paranjpe [50], who observed maturation of MoDC after incubation with PLGA or chitosan films.

These contradictory results may be due to various variables. Firstly, the experimental protocols used in the past studies varied markedly regarding the specific polymer types and quality, the MP size, the source and preparation of MoDC. In the present and our previous study, the 
MoDC were prepared from freshly collected human blood under fully aseptic and serum-free conditions, as currently used in clinical environments [26]. In at least some of the studies by other authors, such precautions were not taken for preparing the MoDC; for example, Jilek et al. [24] used heat-inactivated human serum for MoDC generation. We believe that the lack of DC maturation upon MP incubation is scientifically a much stronger argument than the observation of maturation. Clearly, PLGA MP do not possess any known pathogen-associated molecular patterns (PAMP) that could possibly stimulate maturation (see below). Therefore, DC maturation upon MP incubation is unlikely, unless the working conditions are not fully aseptic. Maturation of DC upon particle incubation might indeed reflect some extent of contamination of the MP themselves or of the cells during preparation.

Under physiological conditions, maturation of DC is induced by pathogens and related molecules like LPS or bacterial DNA [22]. Accordingly, the incorporation of agents mimicking PAMP into biodegradable particulate antigen delivery systems was suggested to enhance the resulting immune response [51]. In this context, we recently proposed the concept of composite PLGA MP coated with cationic polymers such as chitosan [17]. Here, the PLGA core can act as an antigen release-controlling matrix, whereas the cationic polymeric coating is a suitable substrate for an adsorptive or covalent attachment of PAMP-related substances. Such immunostimulating agents encompass, e.g., bacterial DNA containing $\mathrm{CpG}$ motifs [52], double-stranded RNA, its analogue poly(inosinecytidylic acid) (Poly I:C) [53], or specific antibodies $[18,54]$ or peptides. Besides the adsorption of PAMPrelated agents, MP exposing cationic coatings may also offer a template for the complexation of DNA or RNA vaccines. Such polyplexation may physically stabilize nucleic acid therapeutics and preserve them against degradation, especially caused by enzymatic cleavage.

Future studies will focus on the design of MP decorated with bioactive ligands, e.g. PAMP, and nucleic acid vaccines. Such novel MP may have potential for both DC-based immunotherapy and parenteral vaccination. Especially PAMP-decorated MP may pave the way to enhanced immune responses, especially cellular responses.

\section{Conclusions}

Novel PLGA MP types with different polyelectrolyte coatings and surface charge were prepared by a micromixer-based $\mathrm{w} / \mathrm{o} / \mathrm{w}$-solvent extraction/evaporation process. Exposure of the different PLGA MP types to immature monocyte-derived dendritic cells resulted in marked particle uptake by the cells, but no alterations in their phenotype, as assessed by the expression levels of the cell surface molecules CD80, CD83, CD86, and HLA-DR. In addition, the functional cell activities of maturation upon cytokine stimulation, migration towards chemokines, and cytokine release were not altered upon phagocytosis of the different MP. These findings offer promising perspectives for using these MP for ex vivo antigen loading of professional antigen-presenting cells as cellular vaccines. In addition, these cationic MP represent an interesting template for additional MP modification, e.g., by adsorption of additional adjuvants like immunostimulatory $\mathrm{CpG}$ oligodeoxynucleotides.

\section{Acknowledgments}

The authors would like to thank Sandra Hofmann, ETH Zurich, for valuable advice and discussion.

\section{References}

[1] Langer R. New methods of drug delivery. Science 1990;249:1527-33.

[2] Johansen P, Men Y, Merkle HP, Gander B. Revisiting PLA/PLGA microspheres: an analysis of their potential in parenteral vaccination. Eur J Pharm Biopharm 2000;50:129-46.

[3] Morris W, Steinhoff MC, Russell PK. Potential of polymer microencapsulation technology for vaccine innovation. Vaccine 1994; $12: 5-11$

[4] Luzardo-Alvarez A, Blarer N, Peter K, Romero JF, Reymond C, Corradin $\mathrm{G}$, et al. Biodegradable microspheres alone do not stimulate murine macrophages in vitro, but prolong antigen presentation by macrophages in vitro and stimulate a solid immune response in mice. J Control Release 2005;109:62-76.

[5] Thiele L, Rothen-Rutishauser B, Jilek S, Wunderli-Allenspach H, Merkle HP, Walter E. Evaluation of particle uptake in human blood monocyte-derived cells in vitro. Does phagocytosis activity of dendritic cells measure up with macrophages? J Control Release 2001; 76:59-71.

[6] Westwood A, Healey GD, Williamson ED, Eyles JE. Activation of dendritic cells by microparticles containing Bacillus anthracis protective antigen. Vaccine 2005;23:3857-63.

[7] Newman KD, Elamanchili P, Kwon GS, Samuel J. Uptake of poly(D,L-lactic- $c o$-glycolic acid) microspheres by antigen-presenting cells in vivo. J Biomed Mater Res 2002;60:480-6.

[8] Peyre M, Fleck R, Hockley D, Gander B, Sesardic D. In vivo uptake of an experimental microencapsulated diphtheria vaccine following sub-cutaneous immunisation. Vaccine 2004;22:2430-7.

[9] Peyre M, Audran R, Estevez F, Corradin G, Gander B, Sesardic D, et al. Childhood and malaria vaccines combined in biodegradable microspheres produce immunity with synergistic interactions. J Control Release 2004;99:345-55.

[10] Johansen P, Raynaud C, Yang M, Colston MJ, Tascon RE, Lowrie DB. Anti-mycobacterial immunity induced by a single injection of $M$. leprae Hsp65-encoding plasmid DNA in biodegradable microparticles. Immunol Lett 2003;90:81-5.

[11] Men Y, Thomasin C, Merkle HP, Gander B, Corradin G. A single administration of tetanus toxoid in biodegradable microspheres elicits $\mathrm{T}$ cell and antibody responses similar or superior to those obtained with aluminum hydroxide. Vaccine 1995;13:683-9.

[12] Men Y, Gander B, Merkle HP, Corradin G. Induction of sustained and elevated immune responses to weakly immunogenic synthetic malarial peptides by encapsulation in biodegradable polymer microspheres. Vaccine 1996;14:1442-50.

[13] Partidos CD, Vohra P, Jones DH, Farrar GH, Steward MW. Mucosal immunization with a measles virus CTL epitope encapsulated in biodegradable PLG microparticles. J Immunol Methods 1996;195:135-8.

[14] Men Y, Tamber H, Audran R, Gander B, Corradin G. Induction of a cytotoxic $\mathrm{T}$ lymphocyte response by immunization with a malaria specific CTL peptide entrapped in biodegradable polymer microspheres. Vaccine 1997;15:1405-12. 
[15] Audran R, Peter K, Dannull J, Men Y, Scandella E, Groettrup M, et al. Encapsulation of peptides in biodegradable microspheres prolongs their MHC class-I presentation by dendritic cells and macrophages in vitro. Vaccine 2003;21:1250-5.

[16] O'Hagan DT, Singh M. Microparticles as vaccine adjuvants and delivery systems. Expert Rev Vaccines 2003;2:269-83.

[17] Fischer S, Foerg C, Ellenberger S, Merkle HP, Gander B. One-step preparation of polyelectrolyte-coated PLGA microparticles and their functionalization with model ligands. J Control Release 2006;111: $135-44$.

[18] Kempf M, Mandal B, Jilek S, Thiele L, Voros J, Textor M, et al. Improved stimulation of human dendritic cells by receptor engagement with surface-modified microparticles. J Drug Target 2003;11: $11-8$.

[19] O'Hagan DT, Singh M, Dong C, Ugozzoli M, Berger K, Glazer E, et al. Cationic microparticles are a potent delivery system for a $\mathrm{HCV}$ DNA vaccine. Vaccine 2004;23:672-80.

[20] O'Hagan DT, Singh M, Ulmer JB. Microparticles for the delivery of DNA vaccines. Immunol Rev 2004;199:191-200.

[21] Westwood A, Elvin SJ, Healey GD, Williamson ED, Eyles JE. Immunological responses after immunisation of mice with microparticles containing antigen and single stranded RNA (polyuridylic acid). Vaccine 2006;24:1736-43.

[22] Banchereau J, Briere F, Caux C, Davoust J, Lebecque S, Liu YJ, et al. Immunobiology of dendritic cells. Annu Rev Immunol 2000;18:767-811.

[23] Walter E, Dreher D, Kok M, Thiele L, Kiama SG, Gehr P, et al. Hydrophilic poly(D,L-lactide-co-glycolide) microspheres for the delivery of DNA to human-derived macrophages and dendritic cells. J Control Release 2001;76:149-68.

[24] Jilek S, Ulrich M, Merkle HP, Walter E. Composition and surface charge of DNA-loaded microparticles determine maturation and cytokine secretion in human dendritic cells. Pharm Res 2004;21: 1240-7.

[25] Waeckerle-Men Y, Groettrup M. PLGA microspheres for improved antigen delivery to dendritic cells as cellular vaccines. Adv Drug Deliv Rev 2005;57:475-82.

[26] Waeckerle-Men Y, Scandella E, Uetz-Von Allmen E, Ludewig B, Gillessen S, Merkle HP, et al. Phenotype and functional analysis of human monocyte-derived dendritic cells loaded with biodegradable poly(lactide-co-glycolide) microspheres for immunotherapy. J Immunol Methods 2004;287:109-24.

[27] Yoshida M, Babensee JE. Poly(lactic-co-glycolic acid) enhances maturation of human monocyte-derived dendritic cells. J Biomed Mater Res A 2004;71:45-54.

[28] Storni T, Kundig TM, Senti G, Johansen P. Immunity in response to particulate antigen-delivery systems. Adv Drug Deliv Rev 2005;57: 333-55.

[29] Sorimachi K, Akimoto K, Hattori Y, Ieiri T, Niwa A. Secretion of TNF-alpha, IL-8 and nitric oxide by macrophages activated with polyanions, and involvement of interferon-gamma in the regulation of cytokine secretion. Cytokine 1999;11:571-8.

[30] Shibata Y, Honda I, Justice JP, Van Scott MR, Nakamura RM, Myrvik QN. Th1 adjuvant $N$-acetyl-D-glucosamine polymer upregulates Th1 immunity but down-regulates Th2 immunity against a mycobacterial protein (MPB-59) in interleukin-10-knockout and wild-type mice. Infect Immun 2001;69:6123-30.

[31] Peluso G, Petillo O, Ranieri M, Santin M, Ambrosio L, Calabro D, et al. Chitosan-mediated stimulation of macrophage function. Biomaterials 1994;15:1215-20.

[32] Forchhammer L, Thorn M, Met O, Gad M, Weidner MS, Claesson MH. Immunobiological effects of glucosamine in vitro. Scand $\mathbf{J}$ Immunol 2003;58:404-11.

[33] Mori T, Murakami M, Okumura M, Kadosawa T, Uede T, Fujinaga T. Mechanism of macrophage activation by chitin derivatives. J Vet Med Sci 2005;67:51-6.

[34] Strong P, Clark H, Reid K. Intranasal application of chitin microparticles down-regulates symptoms of allergic hypersensitivity to Dermatophagoides pteronyssinus and Aspergillus fumigatus in murine models of allergy. Clin Exp Allergy 2002;32:1794-800.

[35] Freitas S, Walz A, Merkle HP, Gander B. Solvent extraction employing a static micromixer: a simple, robust and versatile technology for the microencapsulation of proteins. J Microencapsulat 2003;20:67-85.

[36] Sallusto F, Lanzavecchia A. Efficient presentation of soluble antigen by cultured human dendritic cells is maintained by granulocyte/ macrophage colony-stimulating factor plus interleukin 4 and downregulated by tumor necrosis factor alpha. J Exp Med 1994;179: 1109-18.

[37] Scandella E, Men Y, Gillessen S, Forster R, Groettrup M. Prostaglandin E2 is a key factor for CCR7 surface expression and migration of monocyte-derived dendritic cells. Blood 2002;100: 1354-61.

[38] Waeckerle-Men Y, Allmen EU, von Moos R, Classon BJ, Scandella E, Schmid HP, et al. Dendritic cells generated from patients with androgen-independent prostate cancer are not impaired in migration and T-cell stimulation. Prostate 2005;64:323-31.

[39] Forster R, Emrich T, Voss C, Lipp M. A general method for screening $\mathrm{mAbs}$ specific for $\mathrm{G}$-protein coupled receptors as exemplified by using epitope tagged BLR1-transfected 293 cells and solidphase cell ELISA. Biochem Biophys Res Commun 1993;196 :1496-503.

[40] Banchereau J, Steinman RM. Dendritic cells and the control of immunity. Nature 1998;392:245-52.

[41] Riol-Blanco L, Sanchez-Sanchez N, Torres A, Tejedor A, Narumiya $\mathrm{S}$, Corbi AL, et al. The chemokine receptor CCR7 activates in dendritic cells two signaling modules that independently regulate chemotaxis and migratory speed. J Immunol 2005;174:4070-80.

[42] Bramwell VW, Perrie Y. Particulate delivery systems for vaccines. Crit Rev Ther Drug Carrier Syst 2005;22:151-214.

[43] Westerfeld N, Zurbriggen R. Peptides delivered by immunostimulating reconstituted influenza virosomes. J Pept Sci 2005;11:707-12.

[44] Vassallo R, Tamada K, Lau JS, Kroening PR, Chen L. Cigarette smoke extract suppresses human dendritic cell function leading to preferential induction of Th-2 priming. J Immunol 2005;175:2684-91.

[45] Macatonia SE, Hosken NA, Litton M, Vieira P, Hsieh CS, Culpepper JA, et al. Dendritic cells produce IL-12 and direct the development of Th1 cells from naive CD4 + T cells. J Immunol 1995;154:5071-9.

[46] de Saint-Vis B, Fugier-Vivier I, Massacrier C, Gaillard C, Vanbervliet B, Ait-Yahia S, et al. The cytokine profile expressed by human dendritic cells is dependent on cell subtype and mode of activation. J Immunol 1998;160:1666-76.

[47] Regnstrom K, Ragnarsson EG, Koping-Hoggard M, Torstensson E, Nyblom H, Artursson P. PEI - a potent, but not harmless, mucosal immuno-stimulator of mixed T-helper cell response and FasLmediated cell death in mice. Gene Ther 2003;10:1575-83.

[48] Sun H, Pollock KG, Brewer JM. Analysis of the role of vaccine adjuvants in modulating dendritic cell activation and antigen presentation in vitro. Vaccine 2003;21:849-55.

[49] Wischke C, Borchert HH, Zimmermann J, Siebenbrodt I, Lorenzen DR. Stable cationic microparticles for enhanced model antigen delivery to dendritic cells. J Control Release 2006;114:359-68.

[50] Babensee JE, Paranjpe A. Differential levels of dendritic cell maturation on different biomaterials used in combination products. J Biomed Mater Res A 2005;74:503-10.

[51] Xie H, Gursel I, Ivins BE, Singh M, O'Hagan DT, Ulmer JB, et al. $\mathrm{CpG}$ oligodeoxynucleotides adsorbed onto polylactide-co-glycolide microparticles improve the immunogenicity and protective activity of the licensed anthrax vaccine. Infect Immun 2005;73:828-33.

[52] Takeshita F, Klinman DM. CpG ODN-mediated regulation of IL-12 p40 transcription. Eur J Immunol 2000;30:1967-76.

[53] Alexopoulou L, Holt AC, Medzhitov R, Flavell RA. Recognition of double-stranded RNA and activation of NF-kappaB by Toll-like receptor 3. Nature 2001;413:732-8.

[54] Cairing J, Barr T, Heath AW. Adjuvanticity of anti-CD40 in vaccine development. Curr Opin Mol Ther 2005;7:73-7. 